\title{
Interprofessional safety reporting and review of adverse events and medication errors in critical care
}

This article was published in the following Dove Medical Press journal:

Therapeutics and Clinical Risk Management

\author{
Claire Chapuis' \\ Sébastien Chanoine ${ }^{1,2}$ \\ Laurence Colombet ${ }^{3}$ \\ Silvia Calvino-Gunther ${ }^{3}$ \\ Caroline Tournegros ${ }^{3}$ \\ Nicolas Terzi ${ }^{2-4}$ \\ Pierrick Bedouch 1,2,5 \\ Carole Schwebel ${ }^{2,3,6}$ \\ 'Pôle Pharmacie, CHU Grenoble \\ Alpes, Grenoble 38000, France; \\ ${ }^{2}$ Université Grenoble Alpes, Grenoble \\ 38000, France; ${ }^{3} \mathrm{CHU}$ Grenoble Alpes, \\ Réanimation Médicale Pôle Urgences \\ Médecine Aiguë, Grenoble 38000, \\ France; ${ }^{4}$ INSERM, UI 042, Université \\ Grenoble-Alpes, HP2, Grenoble \\ 38000, France; ${ }^{5}$ CNRS (UMR5525), \\ TIMC-IMAG, Grenoble 38000, France; \\ 'Inserm U1039 Radiopharmaceutiques \\ Biocliniques, Domaine de la Merci, La \\ Tronche 38700 , France
}

Correspondence: Claire Chapuis Pôle de Pharmacie, CHU Grenoble Alpes, CS 10217, Grenoble 38043, France

Tel +3 3476765497

Fax +3 3476765109

Email cchapuisI@chu-grenoble.fr
Background: The intensive care unit (ICU) environment is prone to the risk of adverse events (AEs) and medication errors (MEs). The objective of this work was to describe a multidisciplinary safety program focused on AE and ME reporting and review in an ICU over a 7-year period.

Methods: The program was implemented in an 18-bed medical ICU of a 2,200-bed university hospital. A multidisciplinary steering committee (intensivist, clinical pharmacist, nurses, and research assistants) met monthly. The first part of the meeting was dedicated to the review of events targeted through an internal voluntary reporting system, and the second part concerned the analysis of the previous month's events, according to a standardized method called Orion, inspired by the aeronautic industry.

Results: A total of 808 AEs were reported, mostly related to medication processes $(30.3 \%$ and $33.4 \%$ for prescription and administration, respectively). Among these, 526 AEs were related to medications (65.1\%), of which 464 were MEs (88.2\%). These MEs concerned mostly anti-infective drugs (23.5\%) and related to wrong doses (35.8\%). Among all AEs reported, 58 (43 MEs [74.1\%]) were analyzed further and found to be associated with anti-infective (16.1\%) and vasoactive drugs (16.1\%). According to National Coordinating Council for Medication Error Reporting and Prevention classification, most AEs caused no harm to patients (category A-D: 38 events, $65.5 \%)$. Nurses were most often involved in the analysis (50.7\%), along with pharmacists (37.5\%). Training was identified as the most frequent corrective action $(45.1 \%)$. Conclusion: This program dedicated to AE and ME reporting, review, and analysis in ICU showed long-term engagement of the health care team in AE surveillance and helped in targeting measures for education, organization, and promoting teamwork and safety.

Keywords: adverse event, medication error, safety, reporting, review, interprofessional

\section{Introduction}

A culture of safety is essential to improve quality of care in intensive care units (ICUs). ${ }^{1,2}$ Because of underlying comorbidities, organ dysfunction, equipment, and polymedication, critically ill patients are at high risk of adverse events (AEs), especially adverse drug events (ADEs). ${ }^{3}$ The World Health Organization defines an $\mathrm{ADE}$ as "any untoward medical occurrence that may present during treatment with a pharmaceutical product but which does not necessarily have a causal relationship with this treatment". ${ }^{4}$ ADEs may also be caused by medication errors (MEs), which the National Coordinating Council for Medication Error Reporting and Prevention (NCC MERP) defines as "any preventable event that may cause or lead to inappropriate medication use or patient harm while the medication is in the control of the health care professional, patient, or consumer". 
Making the complex medication process safer is challenging. ${ }^{5}$ Few errors result in harm, but some occur repeatedly, affecting more patients and increasing risk. A large French study aimed to select medical errors suitable for use as quality indicators using a modified Delphi technique, and then to evaluate the incidence of these medical errors in ICUs and to assess their relationship with mortality. This study, which involved testing a multifaceted program for improving quality of care in ICUs, showed a threefold-increased risk of death in ICU patients experiencing more than two AEs. ${ }^{6}$ Therefore, an effective safety-event reporting and learning system is an essential part of a safety program, in order to avoid patient harm. Important achievements consist mostly in identifying and implementing effective interventions to reduce AEs. ${ }^{7}$

Some methods of event reporting and learning have been previously described and are still used, such as random review of hospital patient records, ${ }^{8}$ morbidity and mortality conferences, ${ }^{9}$ various quality and safety programs, ${ }^{10,11}$ and safety-attitude questionnaires. ${ }^{12}$ Numerous prevention strategies may be considered in the ICU. The implementation of such strategies is highly related to hospital resources (ie, human and financial resources). ${ }^{13}$ Multidisciplinary "experience feedback committees" are devoted to registration, analysis, and correction of precursor events. ${ }^{14,15}$ This concept is followed by high-risk industries, such as aeronautics or nuclear energy, for "near misses" and accidents. Applying such strategies used in high-reliability organizations to health care is a robust approach for analyzing AEs in hospital. Air France Consulting helped the French hospitals in implementing a method named Orion, a systemic analysis based on voluntary reporting, in the analysis of whether AEs caused harm or not. Orion was recommended by the French National Authority for Health. The medical ICU of our hospital chose this method and emphasized the risk related to medications and health products, leading to the implementation of a multidisciplinary steering committee to focus on the therapeutic care process from prescription to administration. The aim of this work was to describe a multidisciplinary collaborative experience focused on therapeutic care process in an ICU over a 7-year period.

\section{Methods}

\section{Setting and participants}

The multidisciplinary program has been in place since 2007 in an 18-bed adult medical ICU of our 2,200-bed tertiary university hospital in Grenoble, France. Computerized prescription order entry (CPOE) has been used in the ICU since June 2011, without review by a pharmacist. Medications have been delivered to the ICU through an automated dispensing system since December 2007. Whenever an AE or ME occurs in an adult ICU patient, the patient or their family is informed of the situation, its consequences, and the immediate corrective actions. AEs and MEs are reported to the members of the multidisciplinary steering committee.

After a period of training by Air France Consulting, the committee met monthly (meetings were interrupted during the summer). This committee was composed of permanent members (ie, an intensivist, a clinical pharmacist, four nurses, and a research assistant) and guest professionals, such as pharmacy technicians, nurse auxiliaries, or students as required. The ICU head staff promoted staff participation. A statement adopting a nonpunitive approach was signed by all members in the committee. The meeting was conducted in a nonjudgmental manner. The standardized Orion method was used for analyzing each event report, based on a systemic approach inspired by the aeronautic industry, as previously explained.

In accordance with Orion, the meeting was divided into two parts (Figure 1). The first part was dedicated to a review of events (AEs and MEs) from an internal voluntary-reporting system, which occurred during the previous month. Any member of the medical, pharmaceutical, or nursing staff could report events, anonymously if desired, via paper or

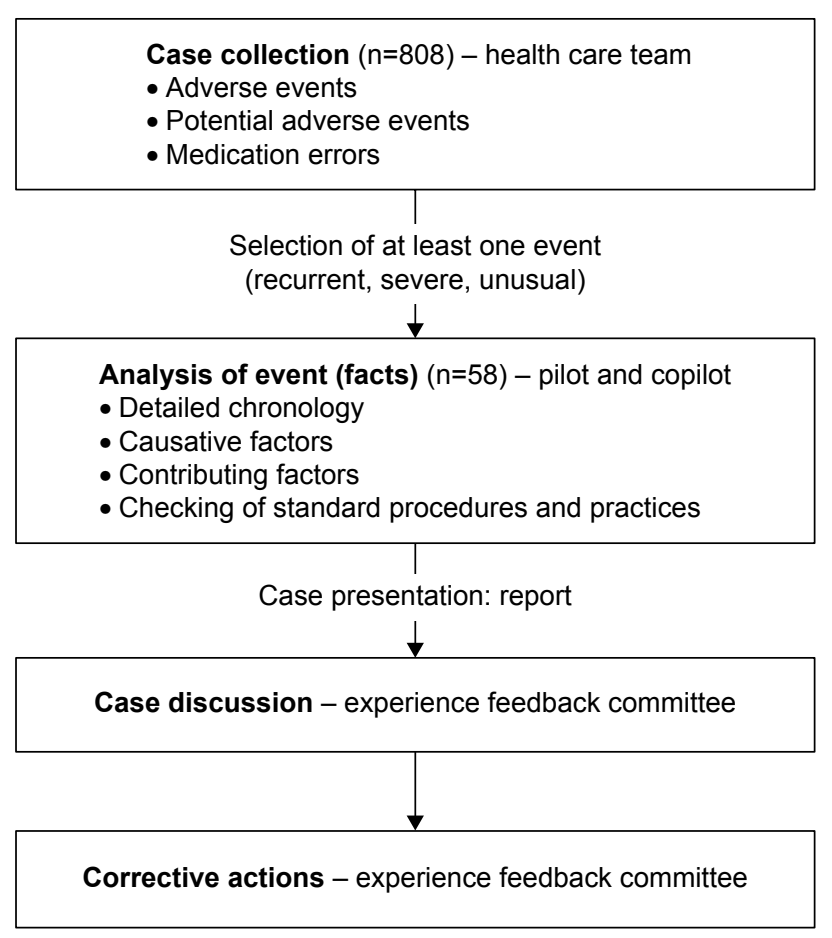

Figure I Procedure for experience feedback committee performance implemented in the intensive care unit of the University Hospital in Grenoble. 
communication to a committee member. Three types of events could be reported: medication process, safety hazards, or unsafe circumstances that could potentially cause harm; near misses or events that could have caused harm to a specific patient, but were caught in time; and AEs - unexpected and undesired outcomes associated with patient care. ${ }^{1}$ The members chose by consensus one relevant event (unusual or potentially harmful) among those reported to be further analyzed by designated "pilot" and "copilot" at the next session. Pilot and copilot were appointed by the group. They could be a physician, pharmacist, nurse or other health care professional, depending on the type of event (eg, a pharmacist and a nurse for an AE concerning medication administration).

The second part of the meeting concerned the analysis of the previous month event by the pilot and copilot. The analysis of each chosen event was conducted following five steps and leading to a written report: event identification, event chronology, root-cause analysis (identification of causes related to political, organizational, and working conditions, team functioning, procedures, actors, and patient), detection of contributing factors (identification of latent and active factors contributing to the genesis of the $\mathrm{AE}$ or $\mathrm{ME}$ ), and checking standard-of-care procedures. Finally, corrective actions were decided collectively by consensus among the committee members before implementation in practice. These corrective actions depended directly on the causes of the $\mathrm{AE}$ or ME. They could be related to guideline writing, organizational changes, communication, training, or resource material. ${ }^{15}$ They were implemented and followed by the committee members who were appointed to them, depending on their area of expertise. Communication was made with all members of the team using email, staff meetings, or posters.

\section{Data collection}

The present analysis was performed for all events reported and analyzed from 2007 to 2013. Data were prospectively collected at each meeting. Each report indicated names and occupations of the participants (physician, nurse, pharmacist, pharmacy technician, pharmacy student, physiologist, research assistant), number and short description of all events, names and work category of the pilot and copilot assigned to the analysis, systematic analysis of the chosen event, and corrective actions. We retrospectively classified the type of event (type of process or product) and the stage in the process (prescription, dispensation, administration, supply chain) for all events, and drug classes (according to the International Classification for Patient Safety and classifications usually used in intensive care studies ${ }^{3,13}$ ) and typology in addition for MEs. For each analyzed event (AE with or without ME), we described report provider, pilot and copilot work category, type of event, stage in the medication process, drug classes, and severity. A multidisciplinary committee (intensivist, two clinical pharmacists, and a pharmacologist) retrospectively assessed ME severity using the NCC MERP method. ${ }^{16}$ Consensus was reached for each case. We distinguished among errors that reach the patient, but cause no harm (NCC MERP categories $\mathrm{C}$ and D); those that cause harm (NCC MERP categories E-H); and those that cause death (NCC MERP category I), and the typology of corrective actions. Among AEs, we analyzed MEs, defined as any preventable event that may have led to inappropriate medication use or patient harm, by their typology and severity.

\section{Results \\ Participants}

From 2007 to 2013, 71 meetings occurred, gathering 7.2 (3 to 14) participants on average each time, as shown in Table 1. As previously described, the permanent members of the multidisciplinary steering committee were an intensivist, a pharmacist (two different pharmacists during the 7 years), four nurses, and a research assistant.

\section{Adverse events reported}

A total of 808 events (11.4/meeting on average) were reported (Table 1). They were mostly related to medication process $(30.3 \%$ and $33.4 \%$ for prescription and administration, respectively). Among them, 526 AEs were related to medications or fluids (65.1\%), of which 464 were MEs. The typology of MEs is presented in Figures 2 and 3. These MEs concerned mostly anti-infective drugs $(23.5 \%)$ and were related to a wrong dose (35.8\%). This was held constant over the 7-year period. We observed a reduction in events related to vasopressors between 2012 and 2013.

\section{Adverse events analyzed}

Among all events reported, 58 (43 MEs [74.1\%]) were analyzed through the standardized Orion method (Table 2). Reporting was performed mostly by nurses as pilots or copilots $(50.7 \%)$, along with pharmacists $(37.5 \%)$. The most common events were associated with anti-infective drugs (16.1\%), vasoactive drugs (16.1\%), and electrolytes (12.5\%). According to the NCC MERP classification, most events caused no harm to patients (categories A-D: 38 events or $65.5 \%$; Figure 4 ). No event with permanent harm (category G) was identified. Five events were reported 
Table I All reported events

\begin{tabular}{|c|c|c|c|c|c|c|c|c|}
\hline & Overall & 2007 & 2008 & 2009 & 2010 & 2011 & 2012 & 2013 \\
\hline Meetings & 71 & 10 & 11 & 10 & 11 & 10 & 8 & 11 \\
\hline Participants per meeting, mean (range) & $7.2(3-14)$ & $7.2(7-12)$ & $6(4-8)$ & $6.5(5-9)$ & $7.8(5-13)$ & $5.6(5-12)$ & $6.3(3-12)$ & $10.4(6-14)$ \\
\hline Events reported & 808 & 55 & 86 & 180 & 93 & 138 & 106 & 150 \\
\hline Events analyzed, $\mathrm{n}(\%)$ & $58(7.2)$ & $7(12.7)$ & $6(7.0)$ & $9(5.0)$ & $10(10.7)$ & $10(7.2)$ & $6(5.7)$ & $10(6.7)$ \\
\hline \multicolumn{9}{|l|}{ Type of incident, $n(\%)$} \\
\hline Clinical process/procedure & $|2|(15.0)$ & 0 & $6(7.0)$ & $11(6.1)$ & $19(20.4)$ & $36(26.1)$ & $31(29.2)$ & $18(12.0)$ \\
\hline Medication/IV fluid & $526(65.1)$ & $46(83.6)$ & $68(79.1)$ & $149(82.8)$ & $61(65.6)$ & $69(50.0)$ & $47(44.3)$ & $86(57.3)$ \\
\hline Medical device/equipment & $86(10.6)$ & $4(7.3)$ & $6(7.0)$ & $4(2.2)$ & $5(5.4)$ & $21(15.3)$ & $15(14.2)$ & $31(20.7)$ \\
\hline Nutrition (including parenteral) & $43(5.3)$ & $5(9.1)$ & $4(4.6)$ & $4(2.2)$ & $4(4.3)$ & $5(3.6)$ & $11(10.4)$ & $10(6.7)$ \\
\hline Blood products & $25(3.1)$ & 0 & 0 & $12(6.7)$ & $3(3.2)$ & $5(3.6)$ & $2(1.9)$ & $3(2.0)$ \\
\hline Oxygen/gas/vapor & $7(0.9)$ & 0 & $2(2.3)$ & 0 & $\mathrm{I}(\mathrm{I} . \mathrm{I})$ & $2(1.4)$ & 0 & $2(1.3)$ \\
\hline \multicolumn{9}{|l|}{ Stage in process, $\mathrm{n}(\%)$} \\
\hline Prescription & $245(30.3)$ & $17(30.9)$ & $22(25.6)$ & $82(45.6)$ & $28(30.1)$ & $4 \mathrm{I}(29.7)$ & $22(20.8)$ & $33(22.0)$ \\
\hline Dispensation & $19(2.4)$ & $2(3.6)$ & $2(2.3)$ & I (0.6) & $8(8.6)$ & $0(0.0)$ & $\mathrm{I}(0.9)$ & $5(3.3)$ \\
\hline Administration & $270(33.4)$ & $24(43.6)$ & $50(58.1)$ & $55(30.6)$ & $30(32.3)$ & $34(24.6)$ & $23(21.7)$ & $54(36.0)$ \\
\hline Supply chain & $165(20.4)$ & $12(21.8)$ & $7(8.1)$ & $39(21.7)$ & $16(17.2)$ & $23(16.7)$ & $28(26.4)$ & $40(26.7)$ \\
\hline Others & $109(13.5)$ & 0 & $5(5.8)$ & $3(1.7)$ & II (II.8) & $40(29.0)$ & $32(30.2)$ & $18(12.0)$ \\
\hline Medication errors, n (\%) & $464(57.4)$ & $32(58.2)$ & $65(75.6)$ & $122(67.8)$ & $61(65.6)$ & $67(48.5)$ & $45(42.4)$ & $72(48.0)$ \\
\hline
\end{tabular}

to the French National Drug Security Agency because they concerned serious adverse effects related to drug administration (treprostinil overdose with flush and headache, intravenous immunoglobulin overdose with acute renal failure and renal replacement-therapy requirement, nitric oxide-inhalation overdose with methemoglobinemia, thrombocytopenia with proton-pump inhibitor, intravenous voriconazole overdose with neurological symptoms). The 58 events were followed by 237 corrective actions, the nature of which is described in Table 2 . They mostly concerned staff training (45.1\%) and organizational management (34.2\%).
Courses were organized (pharmacology and physiology), and informative and attractive posters were displayed in the ward.

\section{Discussion}

This report describes the 7-year involvement of an experience feedback committee in an ICU dedicated to safety-event reporting and review. This is the first transdisciplinary and multiprofessional study performed over a long period. The number of meetings and participants was consistent over time, showing that engagement was well maintained. Reported events were mostly associated with
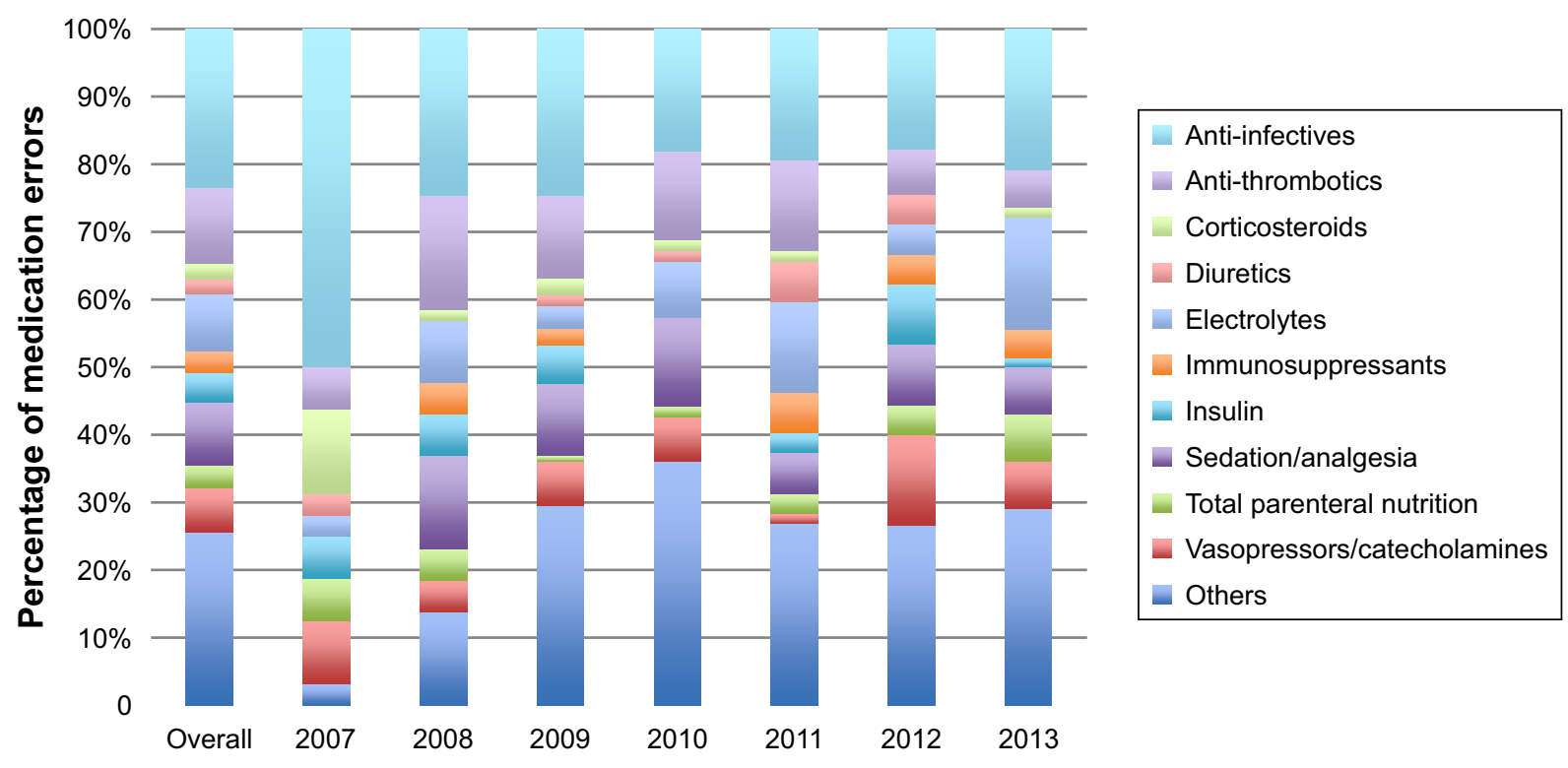

Figure 2 Description of medications related to medication errors reported to the multidisciplinary steering committee from 2007 to 2013 ( $\mathrm{n}=464$ ). Note: Medications were classified according to the main drug classes used in the intensive care unit. 


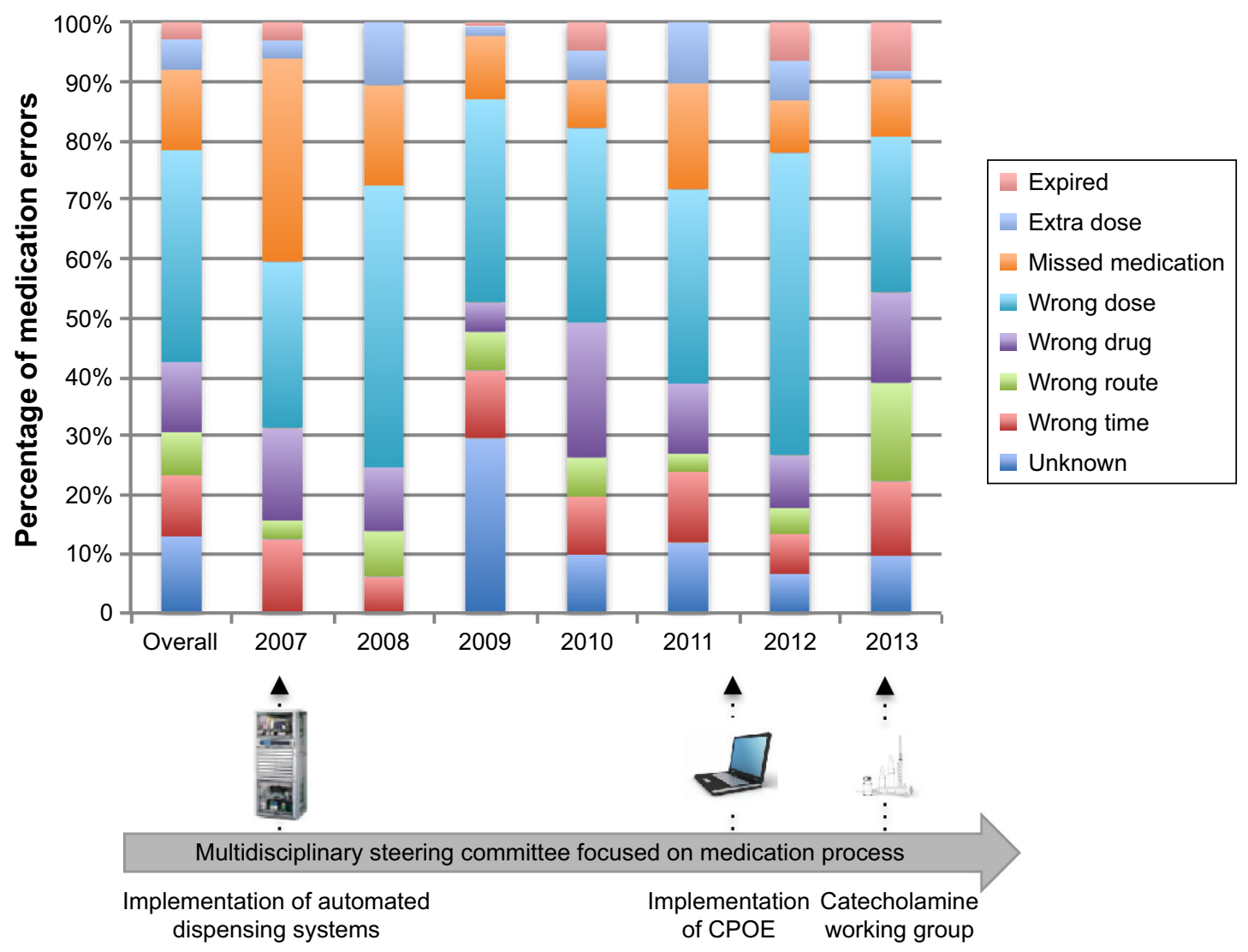

Figure 3 Description of medication-error typologies reported to the multidisciplinary steering committee from 2007 to 2013 ( $n=464$ ).

Note: Main specific corrective actions are described at the bottom of the figure.

Abbreviation: CPOE, computerized prescription-order entry.

medication ordering and administration, which is consistent with previous observations. ${ }^{5,17}$ These events were mostly MEs, mainly not associated with harm. Regarding the type of medications involved in errors, results were similar to other studies in which the most frequently error-related drugs were cardiovascular (24\%-33\%), sedative or analgesic (26\%), antithrombotic (11\%-20\%), and anti-infective (13\%) medications, ${ }^{17}$ and also electrolytes. ${ }^{3}$ Reported-error and $\mathrm{AE}$ rates did not significantly change over time. As events were reported on a voluntary basis, we hypothesized that this depended on many contributing factors, such as staff turnover, which was high for nurses, the culture of safety, and the workload of health care workers. Administration stage was highly influenced by previous stages (ordering and dispensing errors), and administration MEs were often unveiled at the final moment, when the medication was about to reach the patient, or unfortunately already had. Therefore, nurses and pharmacists were strongly involved in reporting events and analyzing them (50\% and $37 \%$, respectively).

Corrective actions arose directly from the root causes highlighted in the analysis. Training activities, such as pharmacology or physiology courses organized by the physicians or the pharmacist, were the most frequent actions. Indeed, in many cases, we identified a lack of knowledge in this field leading to errors. Education is recommended as part of any program intending to reduce AEs and MEs. ${ }^{25}$ Poor communication within the health care team and failures in organization were the second type of frequent issue. Changes in the ward care organization or formalization of roles and responsibilities were proposed. Focusing on potentially dangerous and medication classes used daily, we observed a reduction in events related to vasopressors between 2012 and 2013. Indeed, due to the high number of signaled events, we elaborated new standardized recommendations for the preparation and administration of these specific drugs. Other corrective actions consisted in technological solutions, such as the early implementation of automated dispensing systems in 2007, which can explain the low proportion of reported dispensing errors $(2.4 \%){ }^{18}$ Other technological alternatives, such as CPOE, have been described as important interventions for reducing prescription MEs. ${ }^{19}$ It has also been shown that CPOE can generate 
Table 2 Adverse events analyzed

\begin{tabular}{|c|c|}
\hline & $N=58$ \\
\hline \multicolumn{2}{|l|}{ Type of incident, $n(\%)$} \\
\hline Clinical process/procedure & $3(5.2)$ \\
\hline Medication/intravenous fluid & $46(79.3)$ \\
\hline Oxygen/gas/vapor & $2(3.4)$ \\
\hline Nutrition & $5(8.7)$ \\
\hline Blood products & 0 \\
\hline Medical device/equipment & $2(3.4)$ \\
\hline \multicolumn{2}{|l|}{ Class of drugs, $n(\%)$} \\
\hline Sedation/analgesia & $3(5.4)$ \\
\hline Vasopressors/catecholamines & $9(16.1)$ \\
\hline Antimicrobial & $9(16.1)$ \\
\hline Coagulation-related & $2(3.6)$ \\
\hline Electrolytes & $7(12.5)$ \\
\hline Insulin & $4(7.1)$ \\
\hline Total parenteral nutrition & $4(7.1)$ \\
\hline Others & $18(32.1)$ \\
\hline \multicolumn{2}{|l|}{ Stage in the process, $\mathrm{n}(\%)$} \\
\hline Prescription & $14(25.0)$ \\
\hline Dispensation & $\mathrm{I}(\mathrm{I} .8)$ \\
\hline Administration & $30(53.6)$ \\
\hline Supply chain & $11(19.6)$ \\
\hline \multicolumn{2}{|l|}{ Report provider, $\mathrm{n}(\%)$} \\
\hline Nurse & $40(69.0)$ \\
\hline Physician & II (18.9) \\
\hline Pharmacist & $3(5.2)$ \\
\hline Other & $4(6.9)$ \\
\hline \multicolumn{2}{|l|}{ Pilots/copilots, n (\%) } \\
\hline Nurses & $138(50.7)$ \\
\hline Physicians & $12(4.4)$ \\
\hline $\begin{array}{l}\text { Pharmaceutical staff (pharmacists, } \\
\text { pharmacy technicians, students) }\end{array}$ & $102(37.5)$ \\
\hline Others (physiologist, research assistant) & $20(7.4)$ \\
\hline \multicolumn{2}{|l|}{ Corrective actions, n (\%) } \\
\hline Guideline writing & $20(8.4)$ \\
\hline Organizational changes/communication & $81(34.2)$ \\
\hline Training & $107(45.1)$ \\
\hline Resource material & $29(12.2)$ \\
\hline
\end{tabular}

new types of errors. ${ }^{20}$ In this study, CPOE implementation in 2011 was not associated with a change in events or errors typology. However, technology is not sufficient to avoid MEs. Therefore, systematic reporting and analysis of AEs are important and remain necessary. Event reporting in this experience has proven usefulness in highlighting active failures and latent conditions in ICU organization. This is known as an opportunity for improvment. ${ }^{21}$ Some studies have focused on triggered tools and precursor events, ${ }^{22}$ which could be useful to target some significant events in the $\mathrm{ICU}^{6}$ and may help us improve event reporting in our program.

Other limitations have to be acknowledged in this study. The systemic approach previously developed by high-risk industries regarding near misses and accidents for MEs and AEs occurring in an adult ICU (root-cause analysis) was used. This approach has been criticized. ${ }^{23}$ Even if root-cause analysis has potential value in health care, it can fail to explore real systemic problems contributing to AEs. Corrective actions may be weak and insufficient to decrease event recurrence. We were not able to prove a positive impact of our program on the quality of care in the ICU. Our results also failed to demonstrate a reduction in the number of reported events, because of the weak number of events in each category. High turnover of nursing and medical staff, as usually encountered in ICUs, may explain lack of benefit of corrective actions during follow-up. We showed that this program was a dynamic tool for supervisory staff to address priorities in educational needs for the team. Also, it should be recognized that such organizations as hospitals within health care teamwork have inherent weaknesses and latent failures, such as organization, funding, and policy, which can promote errors. ${ }^{24}$ Some of our corrective actions were related to these issues, and we did not have control over them. There is a need for such resources as financial investment and expertise of specialists in incident investigation. In this work, the team was composed only of health care workers. AEs were voluntarily reported, analyzed, and corrected by physicians, pharmacists, and nurses without any external intervention.

As recommended recently in a large review, prevention strategies for medication-related events have to be applied for each phase within the medication-use process. Active surveillance systems that include reporting, identification, and evaluation were highlighted. ${ }^{25}$ The same review indicated that there was no evidence on the impact of benchmarking for patient safety-surveillance strategies on the rates of MEs, ADEs, or mortality in critically ill patients. ${ }^{25}$ We assume that the kind of program we described here takes part in the culture of safety in an ICU. ${ }^{12,26}$ It also promotes teamwork, ${ }^{27}$ and the multidisciplinary approach is a significant part of a better analysis, leading the different parties to discussions of interest and to learn about medication processes as a whole.

The involvement of the unit head (physicians and nurses) is crucial in achieving a culture of safety. A multidisciplinary and multiprofessional team decision-making process is more efficient to achieve significant improvements in daily procedures and patient safety. Also, on-ward involvement of a 


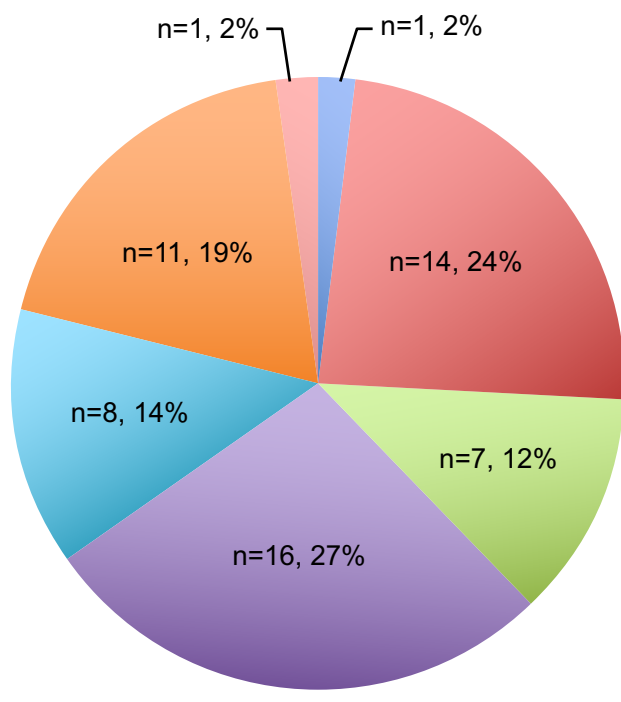

A - No error

B - Error, medication did not reach the patient

C - Error reaches the patient, but no harm

D - Error occurred, no harm, increased monitoring

E - Error harm, need for therapy, temporary harm

F - Error harm, initial or prolonged hospitalization, temporary harm $\mathrm{H}$ - Near-death event

Figure 4 NCC MERP classification (categories A-H) of adverse events analyzed by the multidisciplinary steering committee from 2007 to 2013 ( $n=58$ ).

Note: Category G - Error, permanent harm $=0 \%$.

Abbreviation: NCC MERP, National Coordinating Council for Medication Error Reporting and Prevention.

clinical pharmacist has proven efficient in reducing MEs. ${ }^{28,29}$ We believe that these two factors have been advantageous for this experience.

As stated, unit staff and leadership are critical factors for the success of the program. On the other hand, fear of retribution, confusion about what to report and why, lack of feedback or evidence of action taken following reporting, and inconvenience of paper forms are barriers to reporting. ${ }^{1,30,31}$ We all need to adopt a nonpunitive position to make reporting possible. This is an opportunity to learn from errors.

\section{Conclusion}

This experience feedback committee dedicated to safety reporting and review of AEs and MEs in critical care is one of the recommended active surveillance systems, including reporting, identification, and evaluation. Even if no significant and tangible positive impact can be highlighted, this multidisciplinary patient-centered strategy may be a good tool to drive quality improvement at the unit level, targeting appropriate measures for education, supervision, and organization, promoting teamwork, team performance, and patient safety.

\section{Disclosure}

$\mathrm{SC}$ reports personal fees from AstraZeneca and nonfinancial support from Boehringer Ingelheim, Actelion Pharmaceuticals, and MSD outside the submitted work. NT reports personal fees from Lilly Oncology, Boehringer Ingelheim, and Pfizer outside the submitted work. The other authors report no conflicts of interest in this work.

\section{References}

1. Cochrane D, Taylor A, Miller G, et al. Establishing a provincial patient safety and learning system: pilot project results and lessons learned. Healthc Q. 2009;12(Sp):147-153.

2. Garrouste Orgeas M, Timsit JF, Soufir L, et al. Impact of adverse events on outcomes in intensive care unit patients. Crit Care Med. 2008;36(7):2041-2047. doi:10.1097/CCM.0b013e31817b879c

3. Kane-Gill SL, Kirisci L, Verrico MM, Rothschild JM. Analysis of risk factors for adverse drug events in critically ill patients. Crit Care Med. 2012;40(3):823-828. doi:10.1097/CCM.0b013e318236f473

4. World Health Organization. Safety Monitoring of Medicinal Products. Guidelines for setting up and running a Pharmacovigilance Centre; 2000. Available from: https://www.who.int/medicines/areas/quality_safety/quality_assurance/guidelines/en/. Accessed March 22, 2019.

5. Carayon P, Wetterneck TB, Cartmill R, et al. Characterising the complexity of medication safety using a human factors approach: an observational study in two intensive care units. BMJ Qual Saf. 2014;23(1):56-65. doi:10.1136/bmjqs-2013-001828

6. Garrouste-Orgeas M, Timsit JF, Vesin A, et al. Selected medical errors in the intensive care unit: results of the IATROREF study: parts I and II. Am J Respir Crit Care Med. 2010;181(2):134-142. doi:10.1164/rccm. 200812-1820OC

7. Donaldson L. Reducing harm from radiotherapy. BMJ. 2007;334(7588): 272. doi:10.1136/bmj.39112.454387.BE

8. Heath DA. Random review of hospital patient records. BMJ. 1990; 300(6725):651-652

9. Ksouri H, Balanant PY, Tadié JM, et al. Impact of morbidity and mortality conferences on analysis of mortality and critical events in intensive care practice. Am J Crit Care. 2010;19(2):135-145. doi:10.4037/ ajcc2010590

10. Kernisan LP, Lee SJ, Boscardin WJ, Landefeld CS, Dudley RA. Association between hospital-reported leapfrog safe practices scores and inpatient mortality. JAMA. 2009;301(13):1341-1348. doi:10.1001/ jama.2009.422

11. Stockwell DC, Slonim AD. Quality and safety in the intensive care unit. J Intensive Care Med. 2006;21(4):199-210. doi:10.1177/08850 66606287079

12. Sexton JB, Berenholtz SM, Goeschel CA, et al. Assessing and improving safety climate in a large cohort of intensive care units. Crit Care Med. 2011;39(5):934-939. doi:10.1097/CCM.0b013e318206d26c

13. Patel GP, Kane-Gill SL. Medication error analysis: a systematic approach. Curr Drug Saf. 2010;5(1):2-5. 
14. Lartigau E, Coche-Dequeant B, Dumortier V, et al. Experience feed back committee in radiotherapy (CREX): a compulsory tool for security management evaluation at two years. Cancer Radiother. 2008;12(6-7): 610-613. doi:10.1016/j.canrad.2008.07.007

15. Lecoanet A, Sellier E, Carpentier F, Maignan M, Seigneurin A, François P. Experience feedback committee in emergency medicine: a tool for security management. Emerg Med J. 2014;31(11):894-898. doi:10.1136/ emermed-2013-202767

16. NCCMERP taxonomy of medication error 1998. Available from: http://www.nccmerp.org/taxonomy-medication-errors-now-available. Accessed April 24, 2017.

17. Rothschild JM, Landrigan CP, Cronin JW, et al. The critical care safety study: the incidence and nature of adverse events and serious medical errors in intensive care. Crit Care Med. 2005;33(8):1694-1700.

18. Chapuis C, Roustit M, Bal G, et al. Automated drug dispensing system reduces medication errors in an intensive care setting. Crit Care Med. 2010;38(12):2275-2281. doi:10.1097/CCM.0b013e3181f8569b

19. Bates D, Cohen M, Leape L, Overhage J, Shabot M, Sheridan T. Reducing the frequency of errors in medicine using information technology. J Am Med Inform Assoc. 2001;8(4):299-308.

20. Tamblyn R, Huang A, Kawasumi Y, et al. The development and evaluation of an integrated electronic prescribing and drug management system for primary care. J Am Med Inform Assoc. 2006;13(2):148-159. doi:10.1197/jamia.M1887

21. Moyen E, Camiré E, Stelfox HT. Clinical review: medication errors in critical care. Crit Care. 2008;12(2):208. doi:10.1186/cc6996

22. Rozich JD, Haraden CR, Resar RK. Adverse drug event trigger tool: a practical methodology for measuring medication related harm. Qual Saf Health Care. 2003;12(3):194-200.
23. Peerally MF, Carr S, Waring J, Dixon-Woods M. The problem with root cause analysis. BMJ Qual Saf. 2017;26(5):417-422.

24. Lawton R, McEachan RR, Giles SJ, Sirriyeh R, Watt IS, Wright J. Development of an evidence-based framework of factors contributing to patient safety incidents in hospital settings: a systematic review. BMJ Qual Saf. 2012;21(5):369-380. doi:10.1136/bmjqs-2011-000443

25. Kane-Gill S, Dasta J, Buckley M, et al. Clinical practice guideline: safe medication use in the ICU. Crit Care Med. 2017;45:e877-e915. doi:10.1097/CCM.0000000000002533

26. Pronovost PJ, Berenholtz SM, Goeschel C, et al. Improving patient safety in intensive care units in Michigan. J Crit Care. 2008;23(2): 207-221. doi:10.1016/j.jcrc.2007.09.002

27. Reader TW, Flin R, Mearns K, Cuthbertson BH. Developing a team performance framework for the intensive care unit. Crit Care Med. 2009;37(5):1787-1793. doi:10.1097/CCM.0b013e31819f0451

28. Klopotowska JE, Kuiper R, van Kan HJ, et al. On-ward participation of a hospital pharmacist in a Dutch intensive care unit reduces prescribing errors and related patient harm: an intervention study. Crit Care. 2010; 14(5):R174. doi:10.1186/cc9278

29. Preslaski CR, Lat I, MacLaren R, Poston J. Pharmacist contributions as members of the multidisciplinary ICU team. Chest. 2013;144(5): 1687-1695. doi:10.1378/chest.12-1615

30. Evans SM, Berry JG, Smith BJ, Esterman A. Attitudes and barriers to incident reporting: a collaborative hospital study. Qual Saf Health Care. 2006;15(1):39-43. doi:10.1136/qshc.2004.012559

31. Firth-Cozens J. Barriers to incident reporting. Qual Saf Health Care. 2002;11(1):7.
Therapeutics and Clinical Risk Management

\section{Publish your work in this journal}

Therapeutics and Clinical Risk Management is an international, peerreviewed journal of clinical therapeutics and risk management, focusing on concise rapid reporting of clinical studies in all therapeutic areas, outcomes, safety, and programs for the effective, safe, and sustained use of medicines. This journal is indexed on PubMed Central, CAS,

\section{Dovepress}

EMBase, Scopus and the Elsevier Bibliographic databases. The manuscript management system is completely online and includes a very quick and fair peer-review system, which is all easy to use. Visit http://www.dovepress.com/testimonials.php to read real quotes from published authors. 